\title{
Analysis and Design of an Energy Efficient Differential Coherent Chaos Based System in Additive White Gaussian Noise Environment
}

\author{
Nizar Al Bassam ${ }^{1}$ and Oday D. Jerew ${ }^{2}$ \\ ${ }^{1}$ Middle East College, Muscat, Oman \\ ${ }^{2}$ Asia Pacific International College, Sydney, NSW 2008, Australia \\ Correspondence should be addressed to Nizar Al Bassam; nazarhooby@yahoo.co.uk
}

Received 24 March 2017; Revised 6 June 2017; Accepted 28 June 2017; Published 3 August 2017

Academic Editor: Daesik Hong

Copyright (c) 2017 Nizar Al Bassam and Oday D. Jerew. This is an open access article distributed under the Creative Commons Attribution License, which permits unrestricted use, distribution, and reproduction in any medium, provided the original work is properly cited.

\begin{abstract}
A new differential coherent chaos based scheme is proposed and named as Differential Chaos ON-OFF Keying (DCOOK). The proposed scheme provides reduction in bit energy and better bit error performance at large spreading factor. This is achieved by presenting each transmitted bit by either identical segment or no transmission within same bit duration. The receiver performs simple correlation between the received signal and its delayed version to determine the transmitted information utilizing the low correlation between the noise signals. The bit error rate (BER) performance of the proposed scheme is evaluated analytically using Gaussian Approximation (GA) method and compared with the simulation results. The results show excellent agreement between the derived expression and simulation. Moreover, the BER of DCOOK scheme is compared with the standard chaos systems: Chaos ON-OFF Keying (COOK), Differential Chaos Shift Keying (DCSK), and Correlation Delay Shift Keying (CDSK). The comparison results show that DCOOK scheme can always achieve superior performance compared to COOK and CDSK schemes and even exceeds the performance of DCSK scheme at typical spreading factor values.
\end{abstract}

\section{Introduction}

Unlike pseudo random signals which are limited in number and are periodic, chaotic signals can theoretically produce infinite number of chaotic signals which are nonperiodic. Moreover, chaotic signal has broadband spectrum and has the ability to resist fading. All these properties make them of particular interest in communication systems, specifically in spread spectrum communications.

Chaotic communications systems can be classified into coherent, noncoherent, and differentially coherent systems [1]. To recover the information in coherent systems, chaotic receiver should generate replica copy of the chaotic carrier signals which is used by the transmitter. However, this is difficult to achieve in noisy channels due to nonperiodicity property of the chaos signals. In noncoherent systems, synchronization is not required and the information is decoded by estimating one of the unique signal parameters like a power as in noncoherent Chaos Shift Keying (CSK) scheme [2] or Chaotic ON-OFF Keying (COOK) scheme [3]. However, optimum threshold determination is the major drawback of noncoherent systems which causes a poor performance with the noise existence $[3,4]$.

In differential coherent systems, each information bit is presented by two identical time slots. First time slot is called a reference signal and is used to demodulate the information carrying signal in the second time slot as in Differential Chaos Shift Keying (DCSK) and Correlation Delay Shift Keying (CDSK) [5]. This system design requires a double bit energy and rate. To overcome these drawbacks, many differential coherent systems and their analytical BER performances are proposed and tested in AWGN environment [6-8].

Combining reference signal and information bearing signal in one-time slots using Walsh code is proposed 
in $[9,10]$ and initial condition modulation in [11]. Both schemes improve the spectral efficiency; however, systems require complex receiver design. To enhance bit rate of DCSK systems, information carrying signal is modulated with two information bits as in [12]. In addition, modulation of the reference signal is suggested to double the bit rate in [13]. Furthermore, bit error rate of DCSK scheme is enhanced by using multiple repeated sequences in one duration instead of continuous sample function. On the other hand, the receiver requires moving average calculation each time over one bit duration and the filtered segment is correlated with its delayed version [14]. Efficient utilization of transmitted signal energy is proposed in multicarrierDCSK (MC-DCSK) [15]. The scheme requires narrow band modulators with high degree of accuracy which is complex to design.

In this paper, we introduce a simple and energy efficient differentially coherent scheme: Differential Chaos ON-OFF Keying (DCOOK). In DCOOK scheme, two identical chaotic segments are transmitted for bit "1." On the other hand no transmission is required for the same bit duration for bit " 0 ." Thus, only half bit energy is utilized to compete the performance of standard chaos based system. Our contributions in this work are summarized as follows:

(1) The proposed DCOOK scheme offers the following:

(i) Notable improvement in BER (up to $1.5 \mathrm{~dB}$ ) compared to standard DCSK and CDSK schemes using only half of their bit energy.

(ii) Superior BER performance compared to COOK scheme.

(iii) Double bit rate compared to DCSK scheme.

(2) Theoretical value of BER is estimated using baseband model of the proposed scheme. The expression is verified by simulation in AWGN channel. Clear matching is obtained between the estimated values and simulation results.

The remainder of this paper is organized as follows. Standard chaos based systems including DCSK, CDSK, and COOK are described in Section 2. Section 3 gives an explicit description of the proposed system. Section 4 derives the analytical BER performance. The comparison between the theoretical BER results and the simulation results is presented in Section 5. It also compares the BER performance of the proposed system with the DCSK, CDSK, and COOK schemes. Finally, Section 6 draws the conclusions.

\section{Standard Chaos Based Systems Description}

2.1. DCSK. For each bit of information, the DCSK transmitter outputs a chaotic sequence $x_{i}$ at time instant $i$. Each transmitted signal consists of two sample functions with equal time duration and with length of $M$, where $M$ represents the spreading factor. The first sample function is called reference signal, while the second is called information

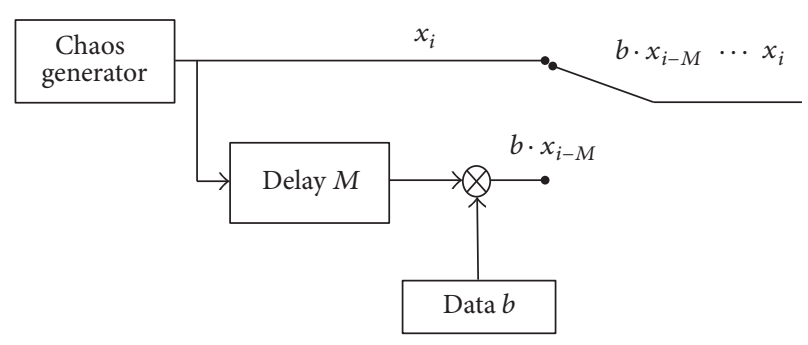

FIGURE 1: DCSK transmitter.

bearing signal. The information bearing is only reference signal delayed by $M$ and multiplied by information bit $b$ where $b \in\{-1,1\}$. The transmitter of DCSK is shown in Figure 1. The transmitted sequence $s_{i}$ for a single bit is given by

$$
s_{i}= \begin{cases}x_{i} & 0<i \leq M, \\ b \cdot x_{i-M} & M<i \leq 2 M .\end{cases}
$$

The average bit energy of DCSK signal can be calculated as

$$
E_{b}=2 M V(x),
$$

where $V(x)$ represents the variance.

The DCSK receiver is shown in Figure 2, and the received signal $r_{i}$ is multiplied by its delayed version. The delay is set to be half bit duration. Thus, the correlator output at the end of bit duration is given by the sum

$$
\begin{aligned}
Z_{\mathrm{DCSK}} & =\sum_{i=1}^{M} r_{i} r_{i-M}=\sum_{i=1}^{M}\left(s_{i}+\zeta_{i}\right)\left(s_{i-M}+\zeta_{i-M}\right) \\
& =b \sum_{i=1}^{M} x_{i}^{2}+\sum_{i=1}^{M} x_{i}\left(\zeta_{i-M}+b \zeta_{i}\right)+\sum_{i=1}^{M} \zeta_{i} \zeta_{i-M}
\end{aligned}
$$

where $\zeta_{i}$ is the Gaussian noise sample such that $E(\zeta)=0$ and variance $V(\zeta)=E\left(\zeta^{2}\right)$. The receiver extracts the information $\widetilde{b}$ according to the following rule:

$$
\widetilde{b}= \begin{cases}1 & Z_{\mathrm{DCSK}} \geq 0, \\ -1 & Z_{\mathrm{DCSK}}<0 .\end{cases}
$$

2.2. CDSK. The CDSK modulated signal is generated by adding the chaotic sequence $x_{i}$ and of the delayed chaotic sequence multiplied by the information signal $b_{\ell}$ as shown in Figure 3. The transmitted signal $s_{i}$ for single bit duration can be written as $s_{i}=x_{i}+b_{\ell} x_{i-M}$ where $\ell$ represents the bit counter.

The CDSK receiver utilizes the low correlation value between chaotic sequence $x_{i}$ and its shifted version $x_{i-M}$ to differentiate between the transmitted information. Hence, average bit energy can be given also by (2). By replacing $M$ by 


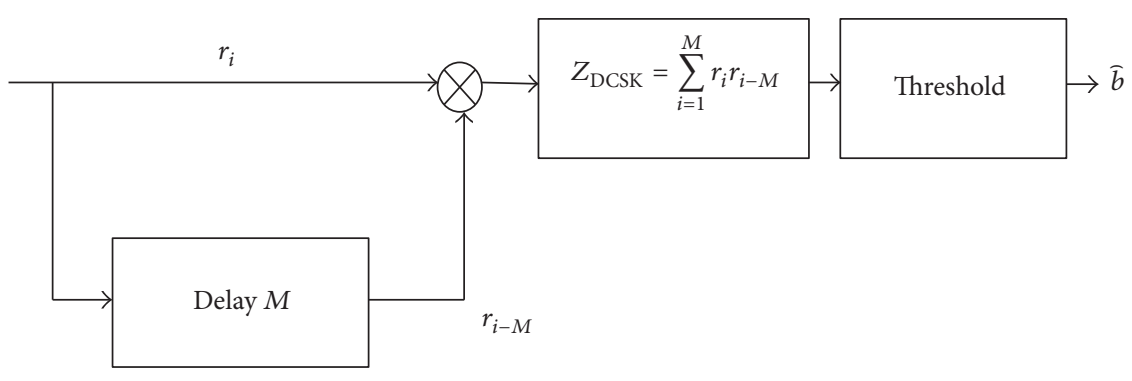

FIGURE 2: DCSK receiver.

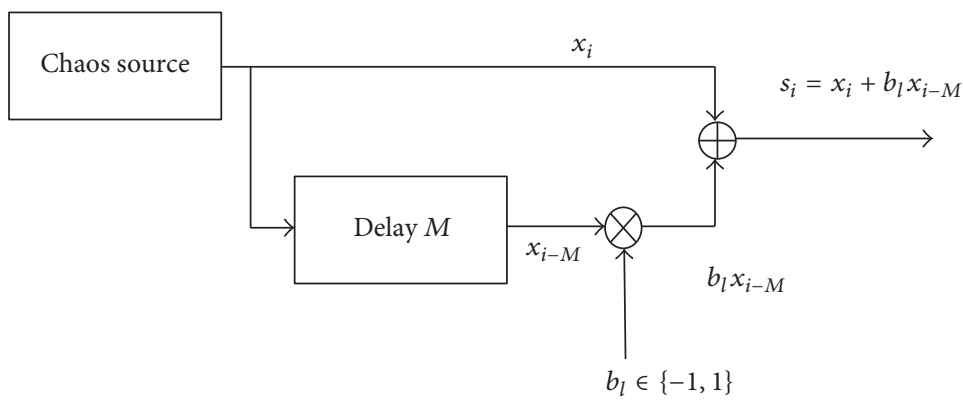

FIGURE 3: CDSK transmitter.

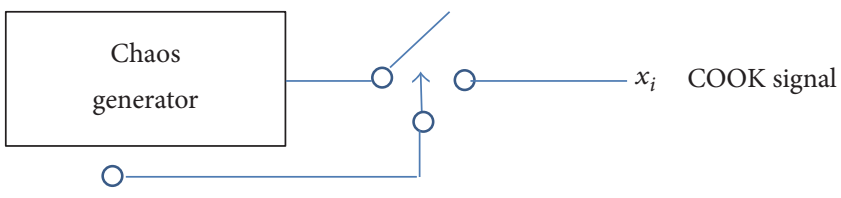

Digital information

to be transmitted

FIGURE 4: COOK transmitter.

$L$, the CDSK receiver becomes similar to the DCSK [5] and the correlator output $Z_{\mathrm{CDSK}}$ can be formulated as

$$
\begin{aligned}
Z_{\mathrm{CDSK}} & =\sum_{i=1}^{M} r_{i} r_{i-M} \\
= & \sum_{i=1}^{M}\left(x_{i}+b_{\ell} x_{i-M}+\zeta_{i}\right)\left(x_{i-M}+b_{\ell-M} x_{i-2 M}+\zeta_{i-M}\right) \\
= & b_{\ell} \sum_{i=1}^{M} x_{i-M}^{2}+\sum_{i=1}^{M} x_{i} x_{i-M}+b_{\ell-M} \sum_{i=1}^{M} x_{i} x_{i-2 M} \\
& +\sum_{i=1}^{M} x_{i} \zeta_{i-M}+b_{\ell} b_{\ell-M} \sum_{i=1}^{M} x_{i-M} x_{i-2 M} \\
& +b_{\ell} \sum_{i=1}^{M} x_{i-M} \zeta_{i_{M}}+\sum_{i=1}^{M} x_{i-M} \zeta_{i}+b_{\ell-M} \sum_{i=1}^{M} x_{i-2 M} \zeta_{i} \\
& +\sum_{i=1}^{M} \zeta_{i} \zeta_{i-M} .
\end{aligned}
$$

Although the systems does not require separate time slot to send the information bearing signal which increases the bit rate, this advantage is defeated by the low BER performance of the system due to multiple intrasignal interference terms in the correlator output.

2.3. COOK. In noncoherent COOK scheme, only one basis function is used as shown in Figure 4. Bit "1" is sent by continuous transmission of the chaotic signal $x_{i}$ within bit duration. Bit " 0 " is presented by null transmission within same duration. The modulation process is simple as controlling turning off and on the chaos generator. Clearly, the bit energy of transmitted signal takes either a positive value or zero depending on the symbol sent.

The receiver structure of COOK scheme is illustrated in Figure 5. Each incoming signal $r_{i}$ is multiplied by itself and averaged over the spreading sequence $M$ which represents simple energy detection process. However, setting threshold at the output is the major disadvantage of the COOK scheme; namely, that threshold value of the decision circuit depends on the noise level. 


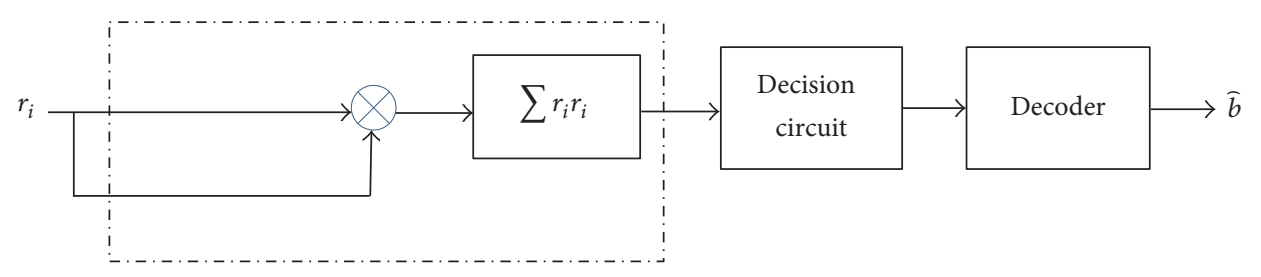

FIGURE 5: COOK receiver.
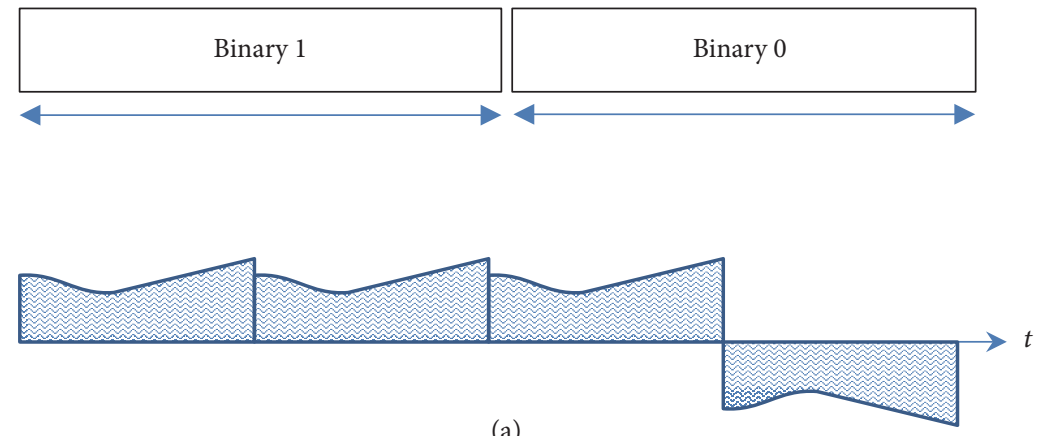

(a)

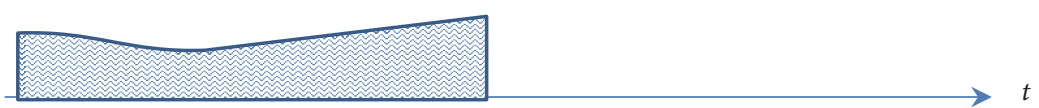

(b)

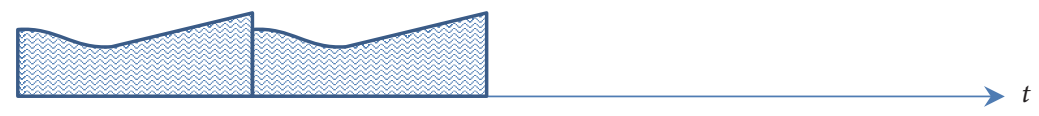

(c)

FIGURE 6: Signal format for chaos based systems. (a) Differentially coherent schemes (CDSK and DCSK). (b) COOK scheme. (c) Proposed scheme (DCOOK).

\section{Proposed System Description}

In this section, signal format of different chaos based systems including the proposed system is given before details of the DCOOK scheme are presented.

3.1. Signal Format. Different signal formats for different chaos based systems are illustrated in Figure 6. In CDSK and DCSK schemes, each bit is transmitted by sending a reference signal followed by information bearing signal. The information bearing signal is selected according to the transmitted bit and is either identical or inverted version from the reference signal as shown in Figure 6(a). The signal format of COOK scheme is shown in Figure 6(b); the transmitter sends one chaotic segment for bit " 1 " and no signal to be sent within the same bit duration for bit " 0 ."

In our system, the source generates two identical, successive chaotic segments for bit "1" as shown in Figure 6(c). For bit " 0 ," the source will stop emitting the chaotic signals for the same bit duration. Hence, average bit energy of DCOOK scheme is reduced to half compared to DCSK and CDSK schemes.

3.2. Transmitter and Receiver Description. In order to generate the signal in Figure 6(c), a baseband modulator is designed as shown in Figure 7, which appears to be a slightly modified version of DCSK transmitter [5].

For bit "1," chaos source is used to generate sample $x_{i}$ at each $i$ th instant within the first half of bit duration. In the next half of bit duration that is after $M$ samples, the switch is connected to the delay element to send the same copy in the next half of the bit duration. For bit " 0 " transmissions, the chaotic source will be disabled and no transmission is required. Hence, the transmitted signal $s_{i}$ at any instant for a single information bit can be formulated as

$$
s_{i}= \begin{cases}b x_{i} & 0<i \leq M, \\ b x_{i-M} & M<i \leq 2 M,\end{cases}
$$

where $b \in\{1,0\}$. 


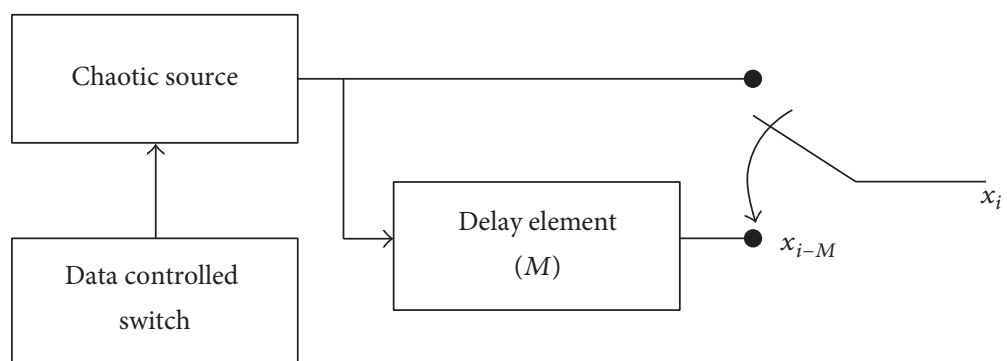

FIGURE 7: DCOOK transmitter.

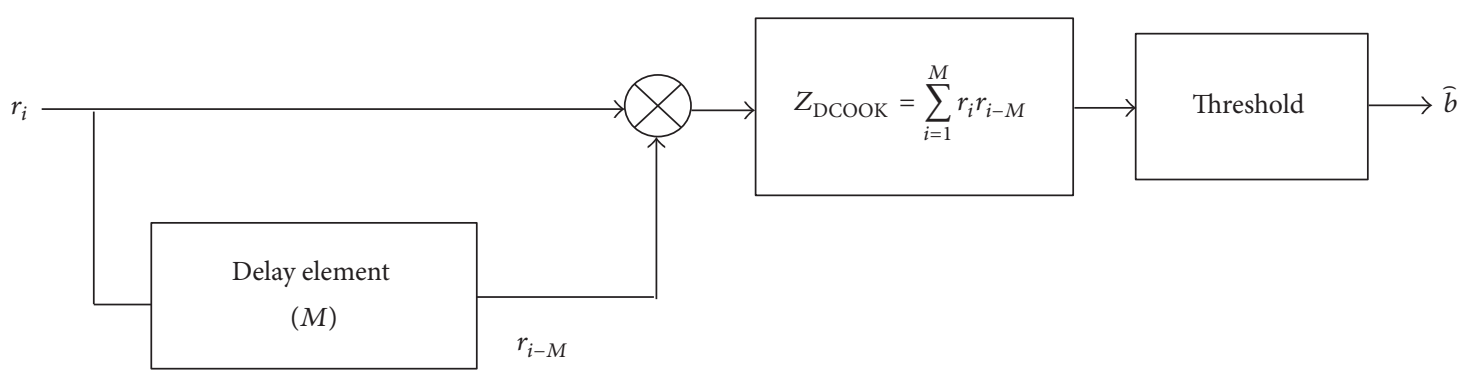

Figure 8: DCOOK receiver.

Figure 8 shows the DCOOK receiver; since coherent receiver requires the synchronized replicas of the chaotic carriers, which is actually quite difficult to achieve [1], differential coherent method is used to recover the information. Under the assumption that transmitter and receiver are synchronized in time, the received signal $r_{i}$ is multiplied by the received signal delayed by $M, r_{i-M}$, and correlator output is averaged over the last half of bit duration. Thus, the correlator output $Z_{\mathrm{DCOOK}}$ can be written as

$$
Z_{\mathrm{DCOOK}}=\sum_{i=1}^{M} r_{i} r_{i-M}
$$

In this paper, the channel under investigation is AWGN, and then the correlator output can be given as

$$
\begin{aligned}
Z_{\mathrm{DCOOK}}= & \sum_{i=1}^{M}\left(s_{i}+\zeta_{i}\right)\left(s_{i-M}+\zeta_{i-M}\right) \\
= & \sum_{i=1}^{M}\left(b x_{i-M}+\zeta_{i}\right)\left(b x_{i-M}+\zeta_{i-M}\right) \\
= & b \sum_{i=1}^{M} x_{i-M}^{2}+b \sum_{i=1}^{M} x_{i-M} \zeta_{i-M}+b \sum_{i=1}^{M} x_{i-M} \zeta_{i} \\
& +\sum_{i=1}^{M} \zeta_{i} \zeta_{i-M} .
\end{aligned}
$$

Analyzing correlator output in (8), it can be easily noticed that there are two different possible outputs according to the value of $b$. The first term in (8) contains the useful signal energy which varies from one bit to another due to chaotic nature. The remaining are the zero-mean disturbance components, which are formed by the noise-signal and noisenoise products. It is important to keep in mind that these components are effective only if $b=1$; otherwise, the correlator input will be only noise-noise term.

Correlator output is decoded by passing the correlator output $Z_{\mathrm{DCOOK}}$ to a threshold detector which operates according to the following rule:

$$
\tilde{b}= \begin{cases}1 & Z_{\mathrm{DCOOK}} \geq \alpha, \\ 0 & Z_{\text {DCOOK }}<\alpha .\end{cases}
$$

The delay element might be challenging for the transceiver integration in our proposed scheme. Fortunately, lots of programmable, picosecond-to-nanosecond-delayed digital delay lines can offer high-data-rate applications. For instance, sampling shift strategies equipped with ultrafast and low-power-consumption sampling techniques and various digital CMOS delay elements can be suggested [12].

\section{BER Performance Evaluation}

The performance of DCOOK scheme is evaluated analytically using Gaussian Approximation (4), which provides an accurate estimation of BER at large value of $M$. For small value of $M$, an integration method is preferred. This is due to the fact that bit energy is not constant for each transmitted bit [16]. The rest of the analyses will be based on the following $[4,5,12]$ :

(1) Symmetric tent map, defined by the equation $x_{n+1}=$ $1-2\left|x_{n}\right|$, is applied to generate the discrete chaotic 
signal. The chaotic sequence is stationary and is uniformly distributed from $(-1,1)$ with zero-mean and computed variance $V(x)=1 / 3$ and $V\left(x^{2}\right)=4 / 45$ [4].

(2) Correlation between each chaotic segment $x_{i}$ and its time shifted version $x_{i+k}$ is for large value of $k$ such that $E\left(x_{i} x_{i+k}\right)=0$.

(3) $\zeta_{i}$ is not only statistically independent from $x_{i}$ for any $(i, j)$, but also statistically independent from $\zeta_{j}$ for any $i \neq j$.

(4) At bit "1," the transmitter sends $2 M$ consecutive $x_{i}$ samples, while for bit "0," null transmission is required. Therefore, average bit energy for DCOOK scheme can be given as $E_{b}=(2 M V(x)+0) / 2=$ $M V(x)$. This is exactly half bit energy for the differentially coherent systems such as DCSK and CDSK.

By applying the previous assumptions and properties, the conditional probability distribution of $\operatorname{Pr}\left(Z_{\mathrm{DCOOK}} \mid b=1\right)$ and $\operatorname{Pr}\left(Z_{\text {DCOOK }} \mid b=0\right)$ can be regarded as Gaussian distributed $[5,17]$. However, average value and variance are different. $Z_{\mathrm{DCOOK}}$ histogram is shown in Figure 9.

Therefore, the performance can be fully characterized by calculating the means and variances as bit " 1 " and bit " 0 " transmission, respectively, as follows:

$$
\begin{aligned}
E\left(Z_{\mathrm{DCOOK}} \mid b=1\right)= & M_{s} V(x)=E_{b} \\
V\left(Z_{\mathrm{DCOOK}} \mid b=1\right)= & \sum_{i=1}^{M} V\left(x_{i-M}^{2}\right) \\
& +\sum_{i=1}^{M} V\left(x_{i-\mathrm{M}}\right) V\left(\zeta_{i-M}\right) \\
& +\sum_{i=1}^{M} V\left(x_{i-M}\right) V\left(\zeta_{i}\right) \\
& +\sum_{i=1}^{M} V\left(\zeta_{i-M}\right) V\left(\zeta_{i}\right) \\
= & \frac{4}{5} M V(x) V(x) \\
& +2 M V(x) V(\zeta) \\
& +M V(\zeta) V(\zeta)
\end{aligned}
$$

Select $V(\zeta)=N_{o} / 2$ where $N_{o}$ is the noise power spectral density, and then (10) can be rewritten as

$$
V\left(Z_{\text {DCOOK }} \mid b=1\right)=\frac{4}{5 M} \frac{E_{b}^{2}}{N_{o}}+E_{b} N_{o}+M \frac{N_{o}^{2}}{4} .
$$

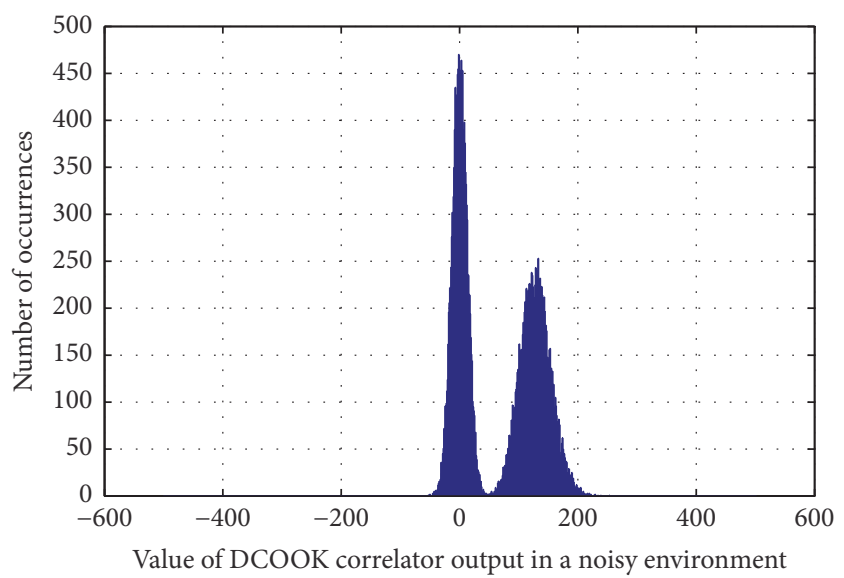

FigURE 9: Histogram of noisy received signal at $E_{b} / N_{o}=16 \mathrm{~dB}$ and $M=100$.

Similarly, we can find

$$
\begin{aligned}
& E\left(Z_{\text {DCOOK }} \mid b=0\right)=0 \\
& V\left(Z_{\text {DCOOK }} \mid b=0\right)=M \frac{N_{o}^{2}}{4} .
\end{aligned}
$$

Assuming $\operatorname{Pr}(0)=\operatorname{Pr}(1)$ and for simplicity, we will chose threshold of the decision circuit in the middle between the average values of the correlator output $\alpha=M V(x) / 2=E_{b} / 2$. Then the average BER can be calculated as

$$
\begin{aligned}
\text { BER } & \\
= & \frac{1}{2} \operatorname{Pr}\left(Z_{\mathrm{DCOOK}} \leq \alpha \mid b=1\right) \\
& +\frac{1}{2} \operatorname{Pr}\left(\mathrm{Z}_{\mathrm{DCOOK}}>\alpha \mid b=0\right) \\
\operatorname{Pr}\left(\mathrm{Z}_{\mathrm{DCOOK}} \leq \alpha \mid b=1\right) & \frac{1}{\sqrt{2 \pi V\left(Z_{\mathrm{DCOOK}} \mid b=1\right)}} \\
& \cdot \int_{-\infty}^{\alpha} e^{-\left(Z_{\mathrm{DCOOK}}-E_{b}\right)^{2} / 2 V\left(Z_{\mathrm{DCOOK}} \mid b=1\right)} \\
= & Q\left(\frac{E_{b}}{N_{o}}\left(\sqrt{\frac{1}{(16 / 5 M)\left(E_{b} / N_{o}\right)+4+M\left(N_{o} / E_{b}\right)}}\right)\right) \\
\operatorname{Pr} & \left(\mathrm{Z}_{\mathrm{DCOOK}}>\alpha \mid b=0\right) \\
= & \frac{1}{\sqrt{2 \pi V\left(Z_{\mathrm{DCOOK}} \mid b=0\right)}} \int_{\alpha}^{\infty} e^{-\left(Z_{\mathrm{DCOOK}}\right)^{2} / 2 V\left(Z_{\mathrm{DCOOK}} \mid b=0\right)} \\
= & Q\left(\frac{E_{b}}{N_{o}} \sqrt{\frac{1}{M}}\right),
\end{aligned}
$$

where $Q(\cdot)$ is the $Q$-Function and is given by

$$
Q(y)=\frac{1}{\sqrt{2 \pi}} \int_{y}^{\infty} e^{-x^{2} / 2} d x .
$$




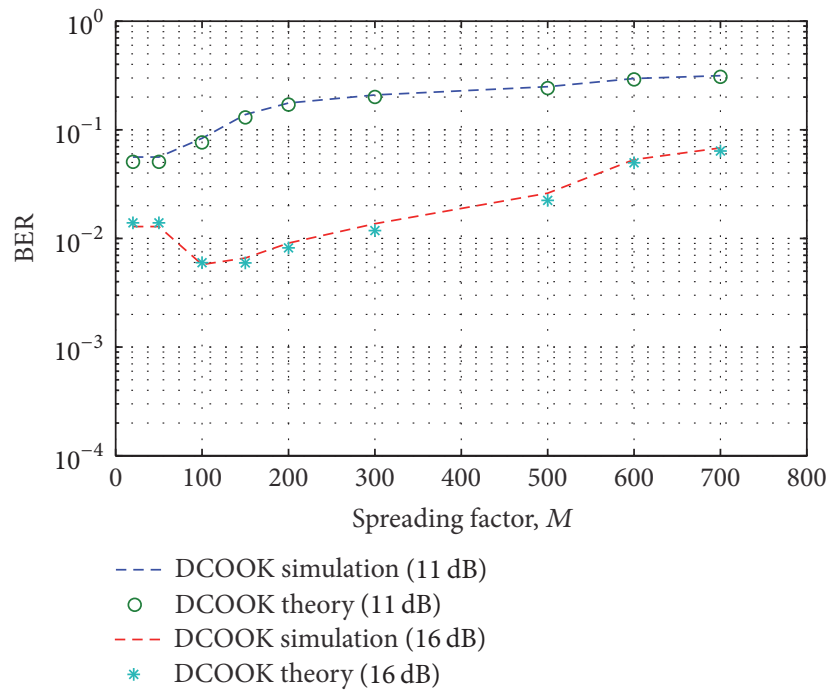

FIGURE 10: BER performance versus spreading factor $M$.

The final expression for BER can be formulated by

BER

$$
\begin{aligned}
& =\frac{1}{2}\left[Q\left(\frac{E_{b}}{N_{o}}\left(\sqrt{\frac{1}{16 E_{b} / 5 M N_{o}+4+M N_{o} / E_{b}}}\right)\right)\right. \\
& \left.+Q\left(\frac{E_{b}}{N_{o}} \sqrt{\frac{1}{M}}\right)\right] .
\end{aligned}
$$

\section{Simulation Results and Discussion}

In this section, the theoretical expression to estimate the BER performance of DCOOK scheme is validated by simulation and with different scenarios. Additionally, the BER performance of DCOOK scheme, after threshold optimization, is compared with different chaos based system in AWGN channel environment.

According to (15), the BER of DCOOK scheme is calculated and validated by simulation. Keep the signal level at $E_{b} / N_{o}=11 \mathrm{~dB}$ and $E_{b} / N_{o}=16 \mathrm{~dB}$ and vary the spreading factor $M$. An excellent matching between the prediction and simulation result as shown in Figure 10 can be noticed. It can also be observed that the proposed scheme has the optimal performance as both curves reach their bottom points at fixed $E_{b} / N_{o}$ with $M$ varying from 10 to 100 . This behavior is caused by the fluctuations in $E_{b}$ at small $M$ and the noisenoise cross correlation at large $M$. On the other hand, the derived equation is used to predict the BER at various $E_{b} / N_{o}$ keeping $M$ constant at $M=100,200,300,500,600$, and 700. An agreement between the predicted value of BER and simulation result can be easily noticed in Figures 11, 12, and 13.

The BER performance of the proposed scheme is tested against the standard DCSK, CDSK, and COOK schemes in AWGN channel environment. Since the correlator output has different energy profiles for 0 and 1 as described earlier,

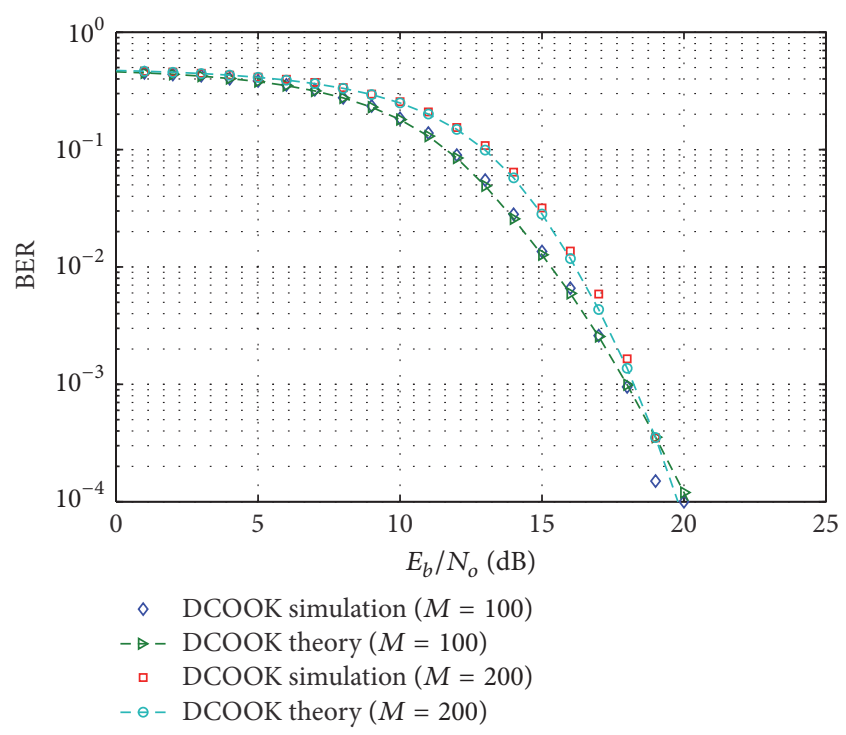

FIGURE 11: Simulated and theoretical BER performance of DCOOK at $M=100$ and $M=200$.

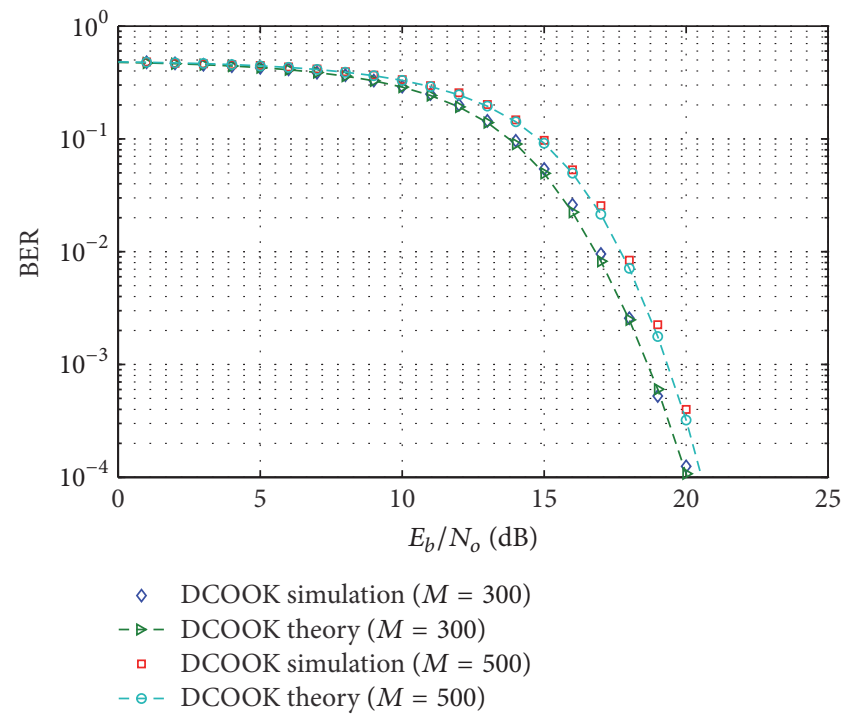

FIGURE 12: Simulated and theoretical BER performance of DCOOK at $M=300$ and $M=500$.

then threshold optimization is required to enhance the performance of DCOOK scheme. One way to choose the optimum threshold is by testing different values of threshold within the range $\left(0, E_{b} / 2\right)$.

At the optimized threshold, DCOOK scheme shows superior BER performance in comparison with the standard COOK scheme as shown in Figures 14, 15, and 16 at $M$ $=500,600$, and 700, respectively. The reason behind this performance is the threshold optimization capability of DCOOK scheme over the COOK scheme. Obviously, in Figure 14, CDSK scheme seems always to be $1.5 \mathrm{~dB}$ worse than DCOOK scheme at BER of $1 \times 10^{-4}$ and this continue for all $M$, the reason of which is that there are much more intrasignal interference terms in the CDSK correlator output. 


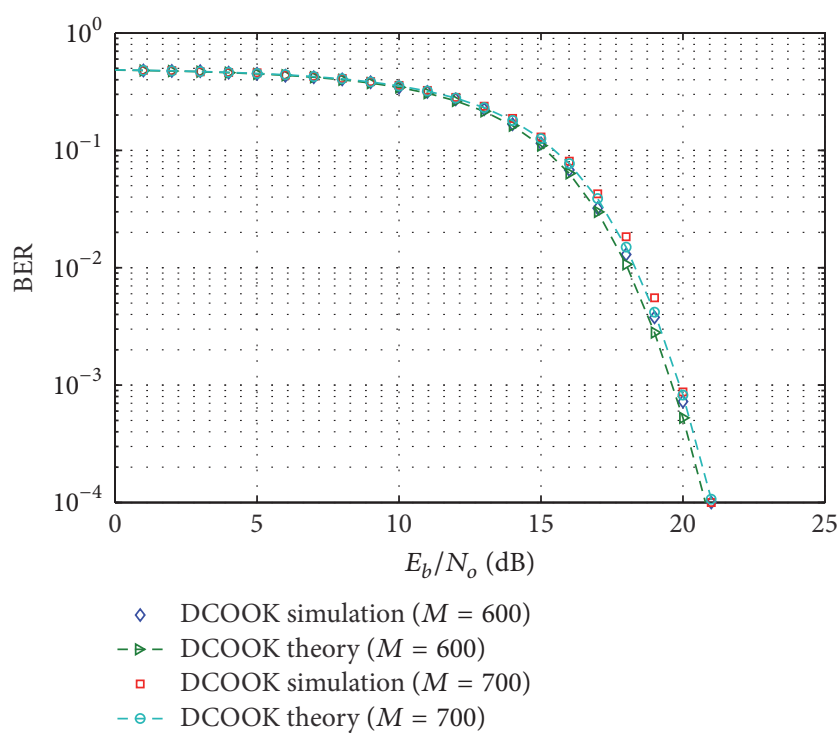

FIGURE 13: Simulated and theoretical BER performance of DCOOK at $M=600$ and $M=700$.

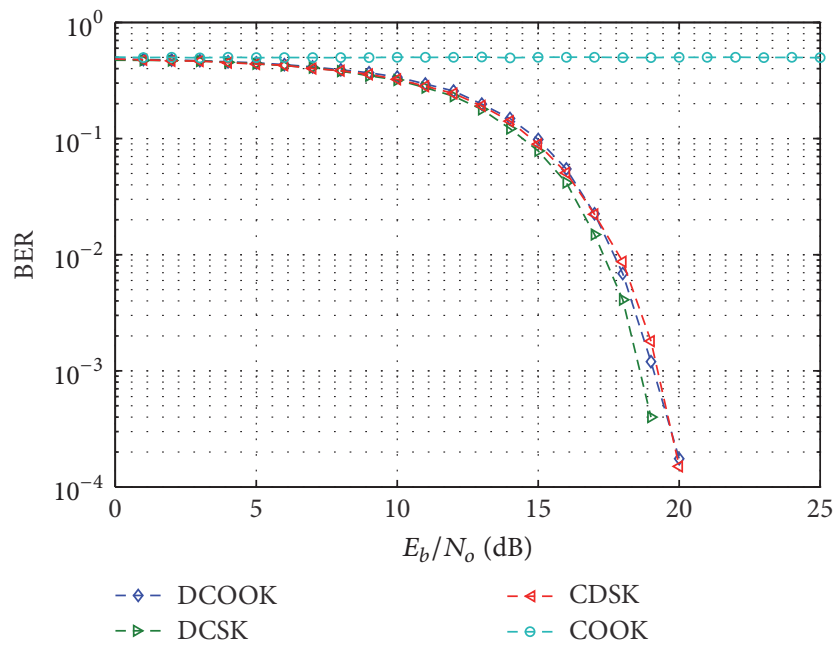

FIGURE 14: BER performance of DCOOK scheme versus COOK, DCSK, and CDSK schemes at $M=500$.

It is interesting to note that DCOOK scheme outperforms the BER performance of DCSK scheme by $1 \mathrm{~dB}$ at $M=600$, while keeping the same performance at $M=500$ and $M=700$ using only half of bit energy used by DCSK scheme. As $M$ increases, BERs for all schemes become worse, which can be attributed to the increasing negative contribution from noisenoise cross terms in their corresponding correlator outputs.

\section{Conclusion}

In this paper, we present a new differential coherent system, DCOOK, which saves bit energy by half and improves BER. Each information bit is presented by sending either two identical chaos signals or no transmission. The performance

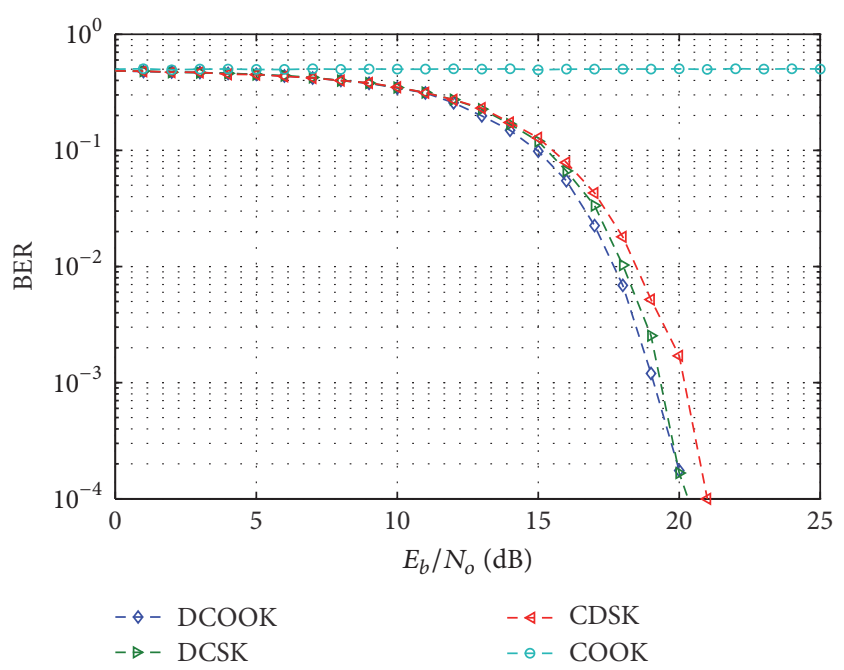

FIGURE 15: BER performance of DCOOK scheme versus COOK, DCSK, and CDSK scheme at $M=600$.

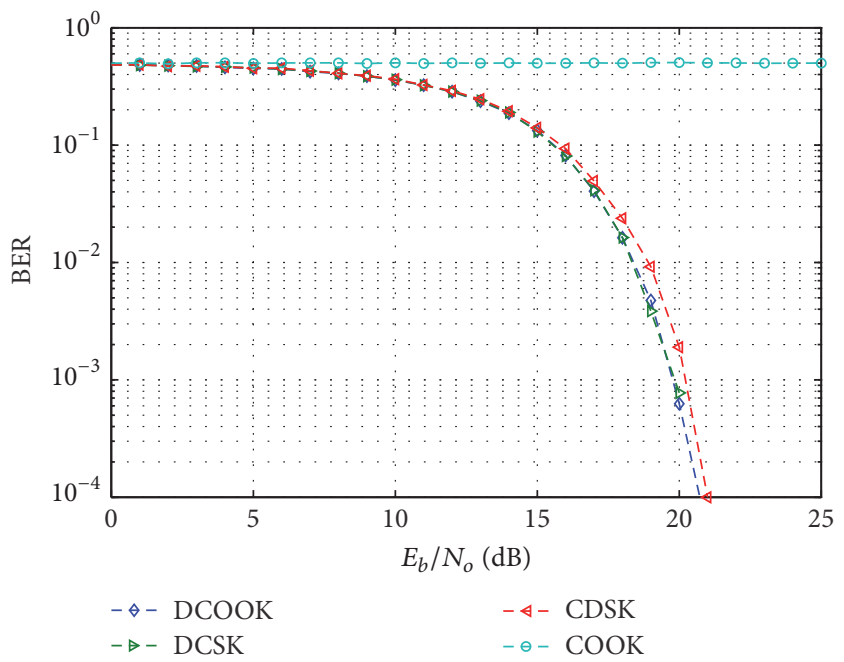

FIGURE 16: BER performance of DCOOK scheme versus COOK, DCSK, and CDSK schemes at $M=700$.

of the proposed scheme is evaluated, and the bit error rate expressions are derived at Gaussian Channel condition. The theoretical BER expression is verified by simulation over a considerable range of spreading factor. Excellent agreement between the derived expression and simulation result is clearly observed. The BER performance of the proposed system is compared to DCSK, CDSK, and COOK schemes.

Our analysis revealed that, at half bit energy, the proposed scheme outperforms DCSK and CDSK at average of $1.25 \mathrm{db}$ while DCOOK shows superior performance compared to $\mathrm{COOK}$ at wide range of spreading factor. Additionally, DCOOK offers double data rate compared to DCSK. Our future work will focus on evaluation of the proposed scheme over the Rayleigh fading channel. 


\section{Conflicts of Interest}

The authors declare that there are no conflicts of interest regarding the publication of this paper.

\section{References}

[1] G. Kolumban, M. P. Kennedy, and L. O. Chua, "The role of synchronization in digital communications using chaos. II. chaotic modulation and chaotic synchronization," IEEE Transactions on Circuits and Systems I: Fundamental Theory and Applications, vol. 45, no. 11, pp. 1129-1140, November 1998.

[2] M. Long, Y. Chen, and F. Peng, "Bit error rate improvement for chaos shift keying chaotic communication systems," IET Communications, vol. 6, no. 16, pp. 2639-2644, 2012.

[3] G. Kis, Z. Jako, M. Kennedy, and G. Kolumban, "Chaotic communications without synchronization," in Proceedings of the International Conference on Telecommunications, IEE, pp. 4953, Edinburgh, UK, 1998.

[4] F. C. Lau and C. K. Tse, "Performance Analysis Methods for Non-Coherent Differential Chaos-Shift-Keying Systems," in Chaos-Based Digital Communication Systems, Signals and Communication Technology, pp. 63-96, Springer Berlin Heidelberg, Berlin, Heidelberg, 2003.

[5] M. Sushchik, L. S. Tsimring, and A. R. Volkovskii, "Performance analysis of correlation-based communication schemes utilizing chaos," IEEE Transactions on Circuits and Systems I: Fundamental Theory and Applications, vol. 47, no. 12, pp. 1684-1691, 2000.

[6] N. Al Bassam and O. Jerew, "Design and implementation of a chaotic scheme in additive white Gaussian noise channel," Journal of Computer Networks and Communications, vol. 2016, Article ID 5976282, 2016.

[7] F. J. Escribano, G. Kaddoum, A. Wagemakers, and P. Giard, "Design of a new differential chaos-shift-keying system for continuous mobility," IEEE Transactions on Communications, vol. 64, no. 5, pp. 2066-2078, 2016.

[8] H. Yang, G.-P. Jiang, and J. Duan, "Phase-separated DCSK: a simple delay-component-free solution for chaotic communications," IEEE Transactions on Circuits and Systems II: Express Briefs, vol. 61, no. 12, pp. 967-971, 2014.

[9] G. Kaddoum and F. Gagnon, "Design of a high-data-rate differential chaos-shift keying system," IEEE Transactions on Circuits and Systems II: Express Briefs, vol. 59, no. 7, pp. 448452, 2012.

[10] W. Xu, L. Wang, and G. Chen, "Performance analysis of the CS-DCSK/BPSK communication system," IEEE Transactions on Circuits and Systems I, vol. 61, no. 9, pp. 2624-2633, 2014.

[11] M. Xu and H. Leung, "A novel high data rate modulation scheme based on chaotic signal separation," IEEE Transactions on Communications, vol. 58, no. 10, pp. 2855-2860, 2010.

[12] H. Yang and G.-P. Jiang, "High-efficiency differential-chaosshift-keying scheme for chaos-based noncoherent communication," IEEE Transactions on Circuits and Systems II: Express Briefs, vol. 59, no. 5, pp. 312-316, 2012.

[13] H. Yang and G.-P. Jiang, "Reference-modulated DCSK: a novel chaotic communication scheme," IEEE Transactions on Circuits and Systems II: Express Briefs, vol. 60, no. 4, pp. 232-236, 2013.

[14] G. Kaddoum and E. Soujeri, "NR-DCSK: a noise reduction differential chaos shift keying system," IEEE Transactions on Circuits and Systems II: Express Briefs, vol. 63, no. 7, pp. 648652, 2016.
[15] G. Kaddoum, F.-D. Richardson, and F. Gagnon, "Design and analysis of a multi-carrier differential chaos shift keying communication system," IEEE Transactions on Communications, vol. 61, no. 8, pp. 3281-3291, 2013.

[16] G. Kaddoum, P. Chargé, D. Roviras, and D. Fournier-Prunaret, "A methodology for bit error rate prediction in chaos-based communication systems," Circuits, Systems, and Signal Processing, vol. 28, no. 6, pp. 925-944, 2009.

[17] N. I. Chernov, "Limit theorems and Markov approximations for chaotic dynamical systems," Probability Theory and Related Fields, vol. 101, no. 3, pp. 321-362, 1995. 


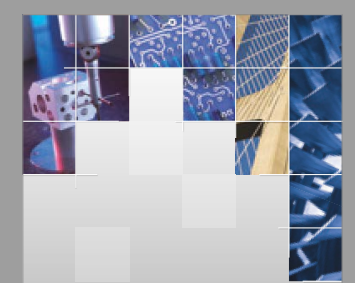

\section{Enfincering}
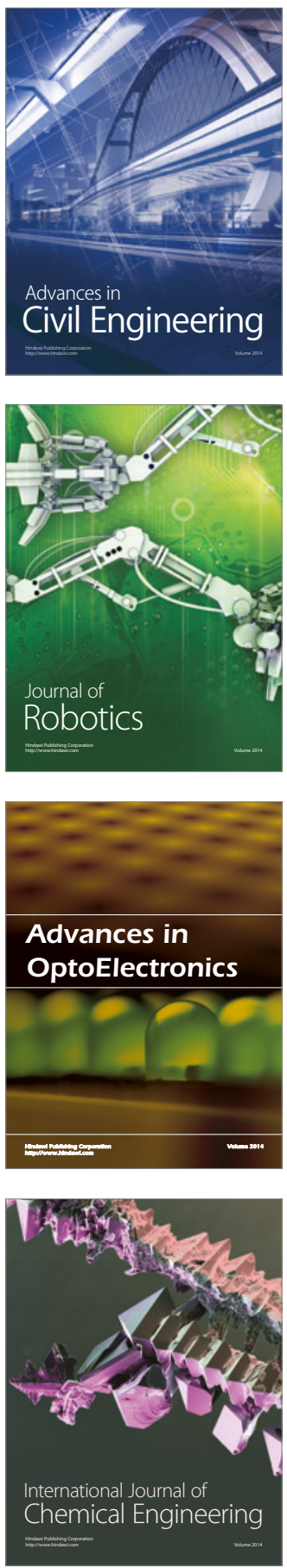

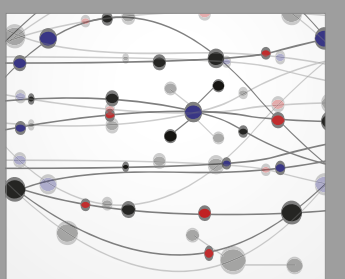

The Scientific World Journal

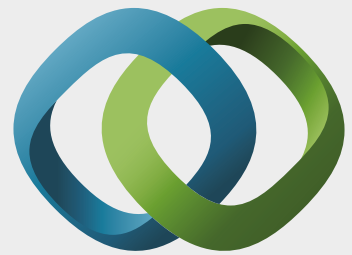

\section{Hindawi}

Submit your manuscripts at

https://www.hindawi.com
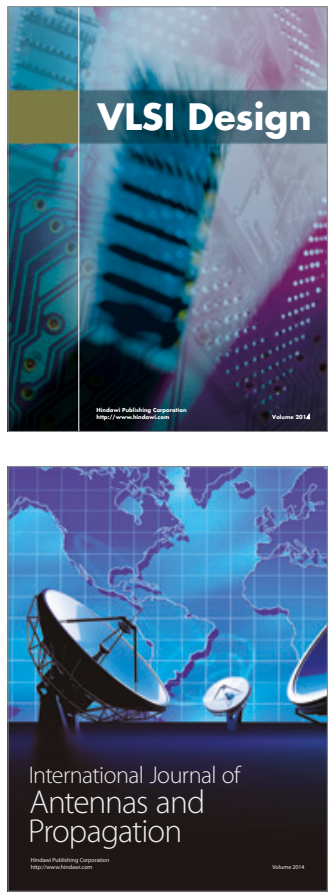

\section{Rotating}

Machinery
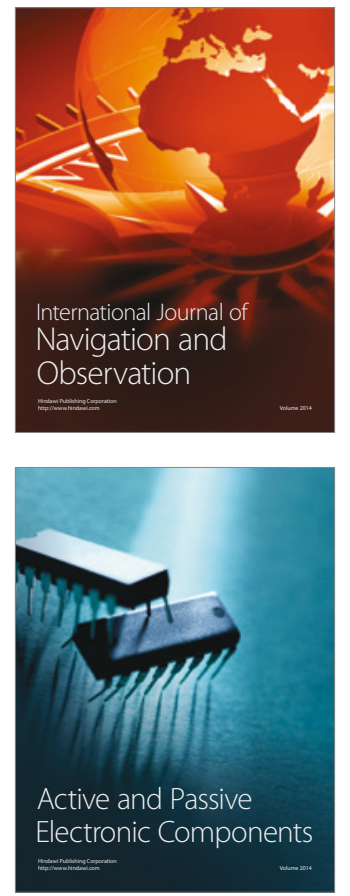
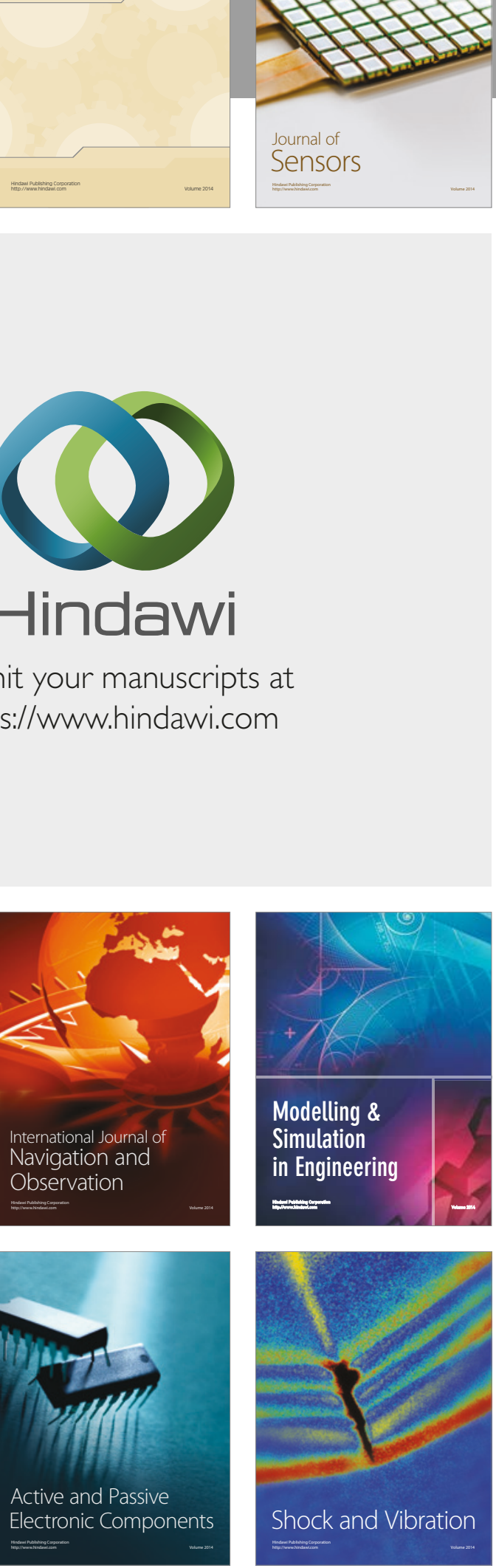
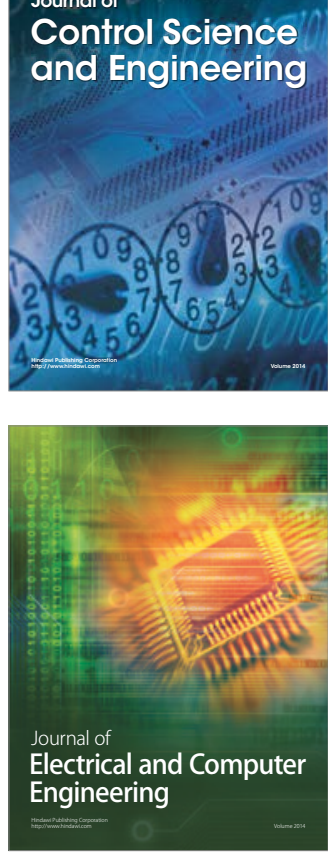

Distributed

Journal of

Control Science

and Engineering
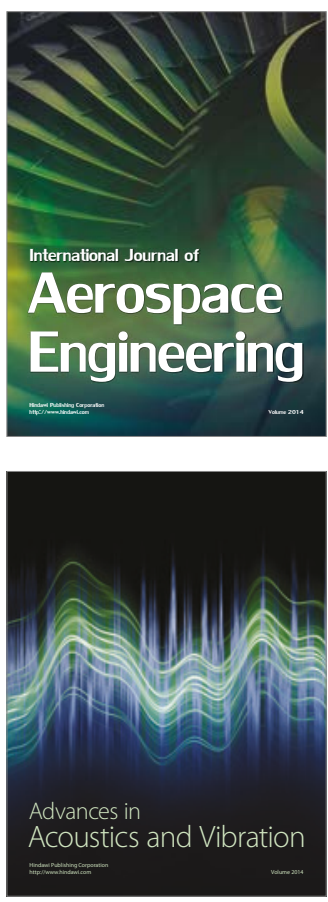

Sensor Networks 